\title{
A Flexible Front-End for Wearable Electrochemical Sensing
}

\author{
Ivan Ny Hanitra ${ }^{* \dagger}$, Lucia Lobello ${ }^{\dagger \dagger}$, Francesca Stradolini ${ }^{\dagger}$, Abuduwaili Tuoheti ${ }^{\ddagger}$, Francesca Criscuolo ${ }^{\dagger}$, Tugba Kilic ${ }^{\dagger}$ \\ Danilo Demarchi $^{\ddagger}$, Sandro Carrara ${ }^{\dagger}$ and Giovanni De Micheli ${ }^{\dagger}$ \\ *Corresponding author: ivan.nyhanitra@epfl.ch \\ $\dagger$ Integrated Systems Laboratory, EPFL, Switzerland \\ $\ddagger$ Department of Electronics and Telecommunications, Politecnico di Torino, Italy
}

\begin{abstract}
This work presents the design and the realization of a flexible front-end circuitry for electrochemical sensing with wearable devices. The hardware combines readout circuitry for amperometric and Open Circuit Potential (OCP) measurements. The sensing platforms are dedicated to lactate and lithium detection in sweat, hence allowing the monitoring of athletes under physical effort. The wearability of the system is ensured by the flexibility of the electronic substrate, its small dimensions that fit an armband case, and the wireless transmission through a Bluetooth Low Energy (BLE) module. The power consumption of the system has been evaluated to be $200 \mathrm{~mW}$, with $3.6 \mathrm{~V}$ on board power supply.
\end{abstract}

\section{INTRODUCTION}

Wearable sensors have received great interest in recent years for personalized diagnosis purposes [1]. Their applications are also valuable for monitoring the physical conditions of an athlete. Indeed, some biological and physiological parameters need to be monitored in order to evaluate fatigue and health status under physical effort. In addition to muscular fatigue, high attention has to be paid on possible symptoms of mental disorders (e.g. meticulous attention to diet, relative hyperactivity), which are widely spread among sport competitors [2]. Endogenous ions [3] and metabolites [4] are relevant to be tracked, given that they provide information about cell growth and metabolism of an organism. Lactate is the basis conjugate of lactic acid, compound produced whenever the human metabolism lacks energy. This is particularly the case under physical efforts [5]. Besides, electrolytes are salient target compounds in monitoring of athletes. Namely, potassium levels are indicators of muscle cramps or dehydration [6], while calcium concentration evidences bone mineral loss [7]. In addition, lithium is monitored for subjects suffering from mental disorders [8]. Indeed, $\mathrm{Li}^{+}$is a mood stabilizer largely adopted by athletes; its medical prescription and dosage is very delicate to ensure safe and effective performance [9]. Therefore, a wearable sensor, able to monitor both metabolites (e.g. lactate) and electrolytes (e.g. lithium) is highly recommended while performing extreme physical exercises [10]. Electrochemical sensors are peculiarly attractive to achieve lactate and $\mathrm{Li}^{+}$sensing since they feature high performance, low cost, rather simple implementation, and above all, portability [11]. Moreover, multi-sensing electrochemical platforms allowing simultaneous monitoring of different compounds of interest have been demonstrated in [12].

Sweat is an optimal fluid for the extraction of biological information since the measure of metabolites and ions in sweat is non-invasive, conversely to blood samples collection, requiring insertion of catheters or an implantable biosensor [13]. It is particularly convenient in sport science applications for which real-time feedbacks to the athlete is suitable. This is feasible since a correlation has been proven between the level of lactate and $\mathrm{Li}^{+}$in blood circulation with the concentration measured in sweat [14]. Lithium monitoring in sweat has been recently proposed in [15]. The electrochemical techniques to detect the aforementioned target compounds are different: (i) amperometry for lactate detection and (ii) potentiometry for $\mathrm{Li}^{+}$sensing. Thus, a combined readout system is required. Some structures have been proposed for multi-channel amperometric sensing: up to five molecules could be monitored in the fully-integrated and implantable platform of [16], while, for example, the portable potentiostat developed in [15] comprises three channels carrying out simultaneous monitoring of anesthetic compounds. The combination of metabolites and electrolytes sensing is achieved in the real-time telemetry system presented in [16], but the thickness $(3 \mathrm{~cm})$ and the rigidity of the substrate of the system are prohibitive for a wearable sensor.

In this work, a four-channel electrochemical platform, manufactured on a flexible substrate, performing both amperometric and potentiometric measurements is presented. The dimensions of the hardware front-end allow its integration in commercially available armband cases. We have also realized and tested in-vitro electrochemical sensors for the detection of lactate and $\mathrm{Li}^{+}$in sweat. The aim of our work is to present the study of an innovative approach for the detection of electrolytes and metabolites with a wearable system. The design and implementation of the analog front-end circuitry is described in section II. Then, the system architecture is discussed in section III. Sensing platforms for lactate and lithium monitoring in sweat are presented in section IV. Finally, conclusions are in section V. 


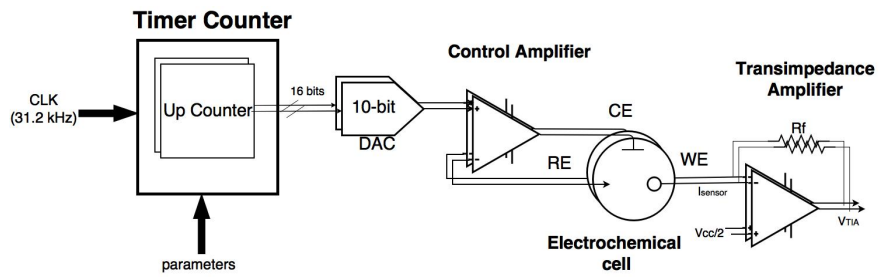

Fig. 1: Two-channels amperometric circuit including waveform generator and transimpedance amplifier.

\section{Circuit ARChitecture}

The analog front-end circuitry implemented for both amperometric and potentiometric sensing is presented in this section. Chronoamperometry (CA), Cyclic Voltammetry (CV), and Differential Pulse Voltammetry (DPV) are the electrochemical detection techniques used for metabolites detection. Two independent sensing channels are in a three-electrode configuration, involving a potentiostat. A signal conditioning block is needed before sampling and processing the output of the sensors. As for the sensing of electrolytes, two independent channels acquire OCP with buffered and differential circuitry.

\section{A. Amperometric sensing}

In amperometric biosensing, a potentiostat allows to control the voltage applied between the Working Electrode (WE) and the Reference Electrode (RE). The sensor outputs a current related to the exchange of electrons at the WE, where the electrochemical reaction occurs. An additional Counter Electrode $(\mathrm{CE})$ is needed to close the current loop. The schematic of the potentiostat is presented in Fig. 1, similar to the work done in [17]. It comprises a waveform generator that outputs a constant voltage, a triangular waveform or ramp up pulses, for CA, CV and DPV, respectively. Triangular waveforms are obtained with a direct digital synthesizer. It is realized with a 16-bit ramp up timer counter and a 10-bit digital-to-analog converter (DAC). The timer counter module of the on board ATxmega32E5 microcontroller is used with a peripheral clock of $31.2 \mathrm{kHz}$. Upwards and downwards staircase voltages, with $3.22 \mathrm{mV}$ step amplitude and variable width are generated. The step width determines the slope of the signal, so that linear ramp-up and ramp-down voltage waveforms of sub- $\mathrm{Hz}$ frequency are obtained. As for DPV pulses, a ramp up timer counter of the same module is used to generate the ramp up pulses. The parameters of the pulses are the amplitude, width and period. The step of the pulses is tuned as well. Signal waveform generated by the timer counter and the DAC are displayed in Fig. 2. DPV pulses with $100 \mathrm{mV}$ step voltage, $300 \mathrm{mV}$ pulse amplitude, $500 \mathrm{~ms}$ pulse width and $1 \mathrm{~s}$ period are represented. CV waveform ramping from 100 to $1100 \mathrm{mV}$, with $200 \mathrm{mV} / \mathrm{s}$ scan rate is shown as well.

An offset of $\frac{V_{c c}}{2}$ is applied at the non-inverting input of the transimpedance amplifier so that the cell voltage $V_{\mathrm{WE}}-\mathrm{V}_{\mathrm{RE}}$ sweeps both positive and negative values. The transimpedance converts the sensor current into a voltage, and the feedback

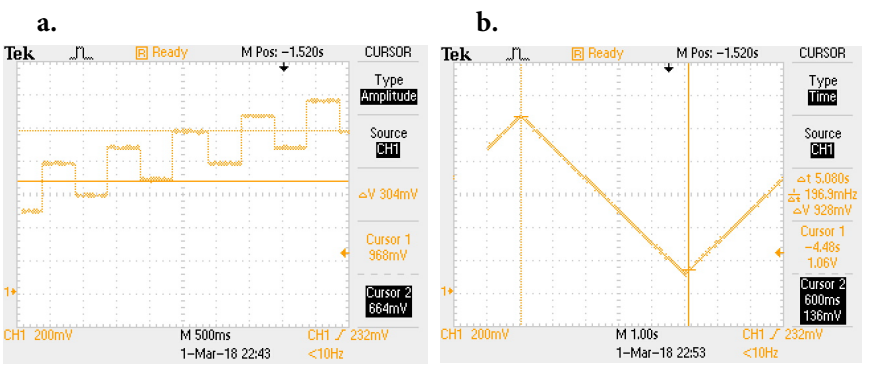

Fig. 2: Signal waveform generated by the timer counter and DAC: a. DPV waveform with $100 \mathrm{mV}$ step voltage, $300 \mathrm{mV}$ pulse amplitude, $500 \mathrm{~ms}$ pulse width and $1 \mathrm{~s}$ pulse period;

b. CV waveform with $100 \mathrm{mV}$ start voltage $1100 \mathrm{mV}$ end voltage and $200 \mathrm{mV} / \mathrm{s}$ scan rate.

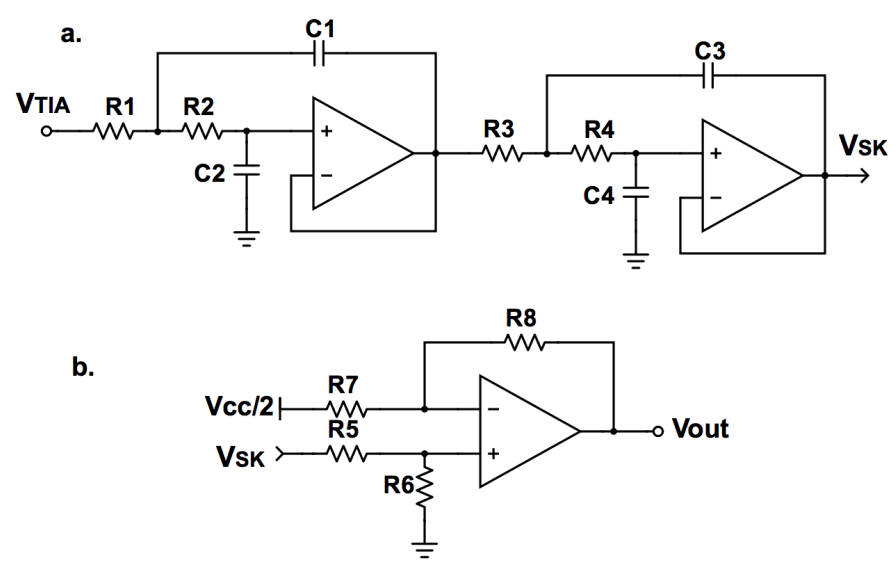

Fig. 3: Signal conditioning block: a. fourth-order Sallen-Key filter. b. output amplifier

resistor sets its gain according to $\mathrm{V}_{\mathrm{TIA}}=\frac{\mathrm{Vcc}}{2}-\mathrm{I}_{\text {sensor }} \cdot \mathrm{R}_{\mathrm{f}}$. Gain of $10 \mathrm{k} \Omega$ and $30 \mathrm{k} \Omega$ are used, so that currents in the range of $[-100 ;+100] \mu \mathrm{A}$ could be sensed.

\section{B. Signal conditioning}

The signal conditioning block is illustrated in Fig. 3. It comprises a fourth-order Sallen-Key low-pass filter (LPF) and an output amplifier. Filtering the high frequency is essential to reduce noise and interference during measurements, and serves as anti-aliasing element. The Sallen-Key topology is an unity gain voltage amplifier with low output impedance. It has been chosen for its high passband gain and high quality factor. The filter implemented is a cascade of two second order LPFs. The filter components are sized with a parametric simulation: the cut-off frequency $\left(\mathrm{f}_{\mathrm{c}}\right)$, the quality factors $\left(\mathrm{Q}_{1}\right.$ and $\mathrm{Q}_{2}$ ), the feedback capacitors and the ratio of capacitors are the parameters. The value of the resistors are output, since there is more flexibility in their choice. The Bode diagram of the Sallen-Key filter is displayed in Fig. 4, featuring an overall $-3 \mathrm{~dB}$ cut-off at $200 \mathrm{~Hz}$. The latter is a good trade-off considering the slow biosignals (sub-Hz signals) and it relaxes components sizing. 

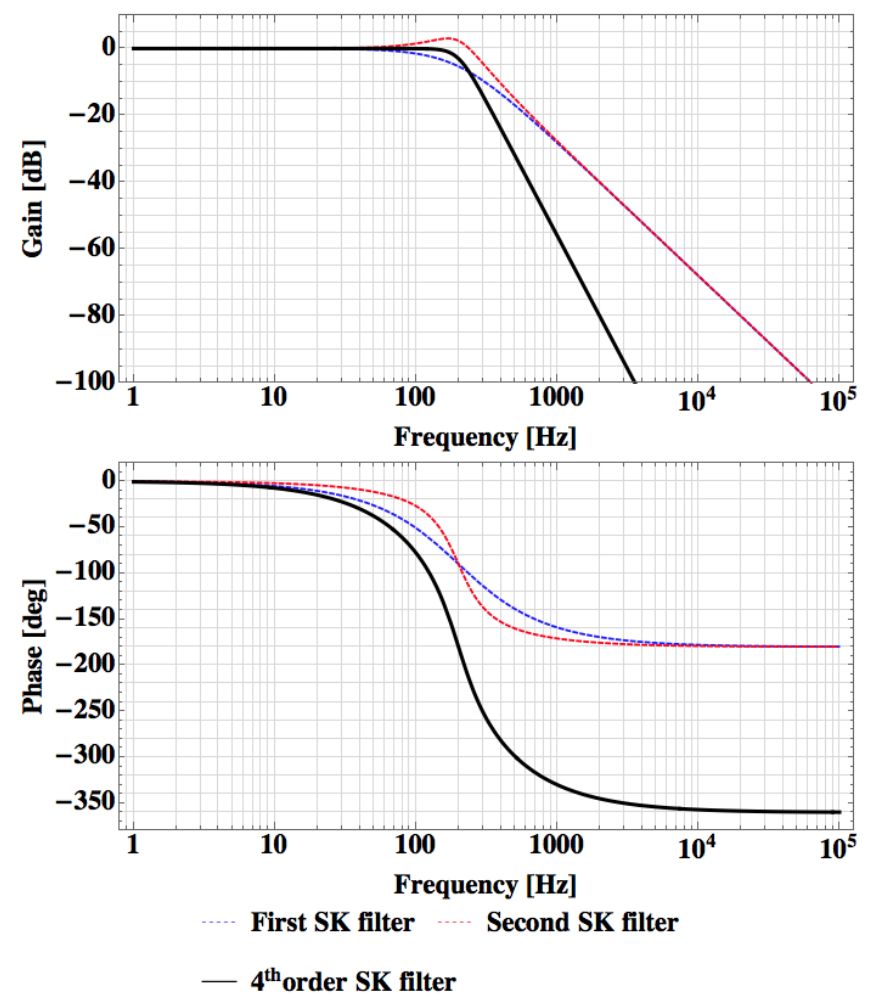

Fig. 4: Bode diagram of the Sallen-Key filter:

$\mathrm{f}_{\mathrm{c}}=200 \mathrm{~Hz}, \mathrm{Q}_{1}=0.541, \mathrm{C}_{1}=3.6 \mathrm{nF}, \frac{\mathrm{C} 2}{\mathrm{C} 1}=0.833$

$\mathrm{Q}_{2}=1.306, \mathrm{C}_{3}=5.6 \mathrm{nF}, \frac{\mathrm{C}_{4}}{\mathrm{C}_{3}}=0.146$

An output amplifier is required to compensate the offset of the transimpedance amplifier. By sizing the resistors properly, the final output voltage is $V_{\text {out }}=1 \mathrm{~V}-I_{\text {sensor }} \cdot R_{f}$.

\section{Potentiometric sensing}

Two independent channels are dedicated to electrolytes sensing. It is achieved by OCP measurements between the sensing electrode and a reference electrode that has a stable potential. Open circuit condition implies that no current should flow through the electrochemical cell [18]. Nevertheless, a small polarization current of few $\mathrm{nA}$ is needed to lower the limit of detection, increase sensitivity and reproductibility of the sensor [19]. This sensor isolation is critical since it induces potential drift. Therefore, a voltage buffer of high input impedance is connected at each terminal of the cell, as illustrated in Fig. 5. MAX44242 buffers with up to $0.5 \mathrm{pA}$ bias current are used. The next stage is a differential amplifier with a gain of 3.9. The signal is amplified utmost so as to use the whole dynamic range of the further analog-to-digital converter (ADC). Thus, OCP in $[0 ; 500] \mathrm{mV}$ range can be resolved. The signal $\mathrm{V}_{\mathrm{DA}}$ is filtered with a fourth-order Sallen-Key filter similar to the one described previously.

\section{System ARChitecture}

The overall system consists of the electrochemical sensor, the Flexible Printed Circuit Board (FPCB), and a terminal

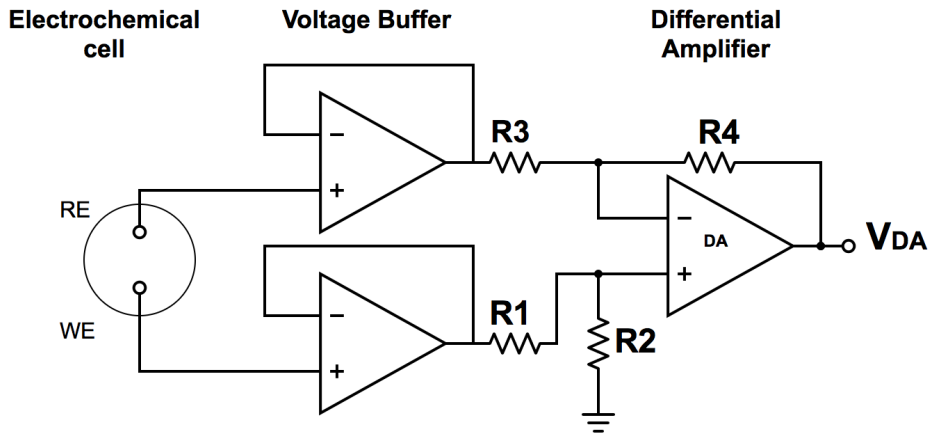

Fig. 5: Analog front-end for OCP readout.

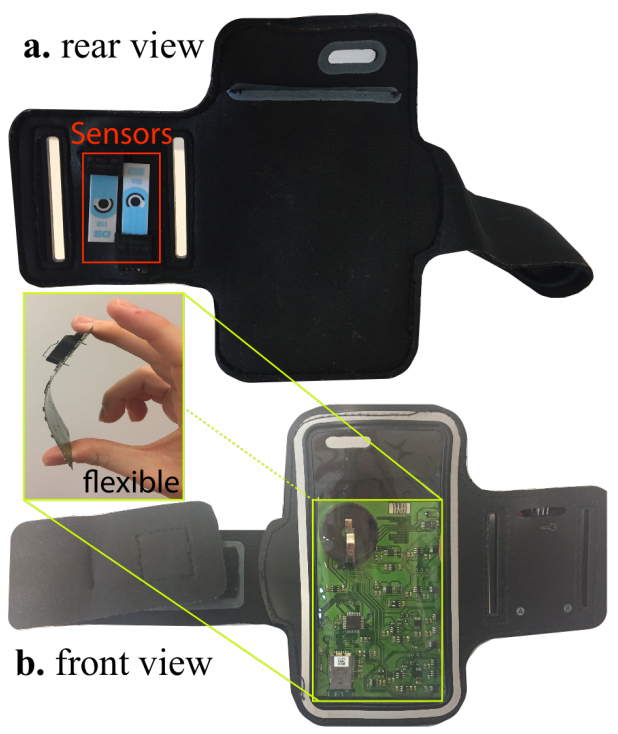

Fig. 6: Integration of the electrochemical platform in an armband: a. rear view $\mathbf{b}$. front view.

controlling the platform. The sensors used are Screen Printed Electrodes (SPEs) that are connected to the hardware frontend with card-edge connectors. The FPCB implements both amperometric and potentiometric readout, and transmits the biological data to a Graphical User Interface (GUI), through a Bluetooth Low Energy (BLE) link. The interface sets the parameters for the measurements as well. The system is tested in laboratory, but it is intended to be integrated in an armband where sweat is collected directly from the user, as it is illustrated in Fig. 6.

\section{A. Flexible Printed Circuit Board}

The FPCB is manufactured with a $0.1 \mathrm{~mm}$ Kapton (polymide) substrate. This material is stable across a wide range of temperatures, has good dielectric properties, and offers mechanical flexibility to the platform. The platform has a size of $97 \times 66 \mathrm{~mm}$, but it could be further shrank by removing all probe points placed after each analog block. Besides, placement and routing could be optimized. Trace 


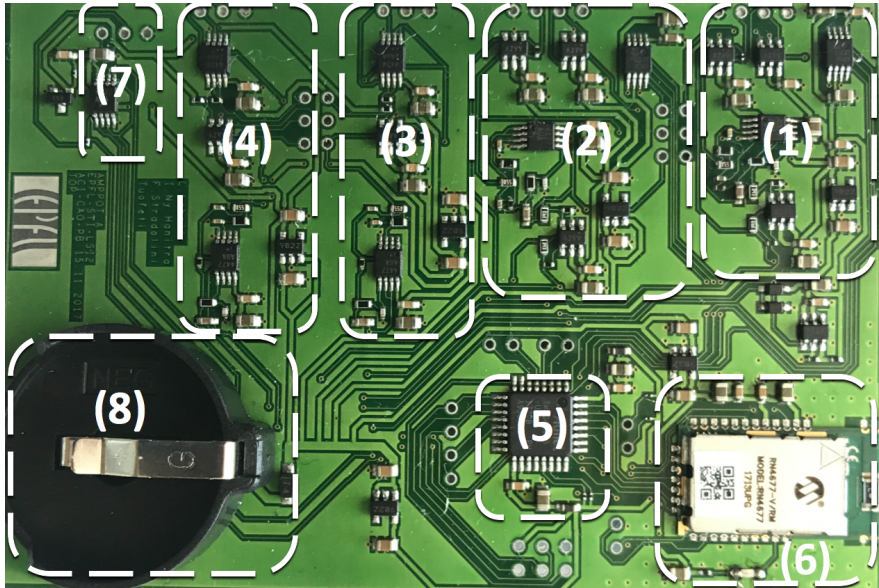

Fig. 7: Different blocks of the FPCB: (1) and (2) are the channels for amperometric readout, while (3) and (4) are the channels for OCP measurement. (5) is the microcontroller unit, (6) is the Bluetooth module, (7) is the UART output module for RS-232 communication and (8) is the power battery holder.

widths of $150 \mu \mathrm{m}$ have been used, and the spacing between signal traces could be reduced, but at a cost of crosstalk.

The FPCB consists of different modules which are listed and highlighted in Fig. 7. A low power ATxmega32E5 microcontroller unit (MCU) is used at the core of the system. Two 16-bit Timer Counters with peripheral clock of $32.5 \mathrm{kHz}$ allow generating the stimulus waveforms for amperometric measurements. The MCU comprises a 12-bit resolution ADC that can sample up to 300 samples per seconds. It is calibrated beforehand so that offset and gain correction coefficients could be computed for better accuracy. Averaging, oversampling and median computation are implemented to increase the resolution of the conversion up to 16 bits. The serial communication capabilities of the MCU are used to relay the signals either to the Bluetooth transceiver or to the UART output module. The latter interfaces the FPCB with the GUI via a RS-232 cable. The board is powered with a lithium rechargeable coin battery $(24 \mathrm{~mm} \varnothing, 7.7 \mathrm{~mm}$ height $)$ of $3.6 \mathrm{~V}$ nominal voltage and $180 \mathrm{mAh}$ capacity. The power consumption of the system is evaluated to be of $200 \mathrm{~mW}$ in normal mode.

\section{B. GUI controlling the platform}

The sensing channels 1 and 2 are dedicated to amperometric sensing, implementing CV, CA and DPV techniques, whereas channels 3 and 4 are used for OCP measurements. The channels are multiplexed. A GUI is developed in Matlab to control the platform. Its different functions are displayed in Fig. 8, where the user is able to:

1) select the sensor to activate

2) set the constant voltage for CA measurement

3) set the parameters for CV measurements (start voltage, end voltage and scan rate)

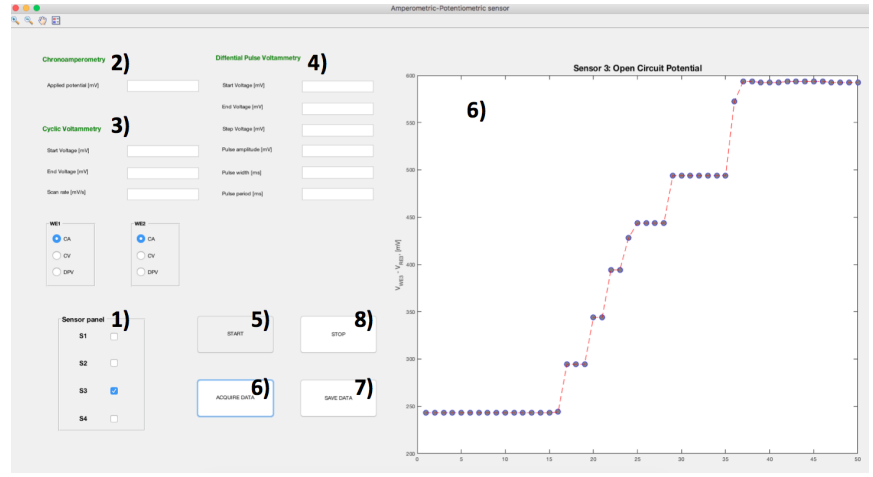

Fig. 8: GUI setting up the FPCB and collecting sensor data. Here, increasing potential differences are applied with a voltage source between WE and RE of the channel 3, performing OCP readout.

4) set the parameters for DPV measurements (start voltage, end voltage, step voltage, pulse amplitude, pulse width, pulse period)

5) request the selected channel to start measurement

6) request the transmission of data, and plotting them in real-time

7) save the received data on a separate Excel sheet for further processing

8) stop the measurement

\section{Wireless transmission}

The communication between the GUI and the FPCB is done through the serial port of the controlling terminal and the one of the MCU. For the initial board validation, an USB to RS-232 serial UART converter cable is connected from the terminal to the UART module on the FPCB. The latter is a level shifter and inverter that converts RS-232 levels into $[0 ; 3.3] \mathrm{V}$, that are the voltage levels of the MCU. It also limits the current output by the host computer.

The dual mode RN4677 Bluetooth is used to establish a wireless communication between the FPCB and the GUI. The Bluetooth transceiver features low energy serial data connectivity through a transparent UART profile. Namely, when the RN4677 is connected and paired to a terminal or device featuring BLE connectivity, it acts as a data pipe between the on board MCU and the terminal, emulating a standard UART communication. The serial communication is done at 115200 baud rate, with no data flow control. The communication protocol implements start tag and stop tag, ensuring safe data transmission. When a request is made by the user, a configuration byte is sent, asking either to start a measurement, send the data in form of packets, pause/resume transmission of data, or stop a measurement. The selected electrode and electrochemical technique are included in the aforementioned configuration byte. 


\section{EleCtrochemicAl MEASUREMENTS}

An Autolab potentiostat driven by Nova 1.11 software has been used to perform preliminary tests on the electrochemical sensors realized for lactate and $\mathrm{Li}^{+}$sensing.

\section{A. Materials and Methods}

1) Chemicals: Lactate Oxidase (LDO) was purchased from Roche (Switzerland), Nafion perfluorinated resin solution 5 wt \%, Lithium Lactate (95\%) and all the others compounds were provided by Sigma-Aldrich (Switzerland). Phosphate Buffered Saline (PBS, $10 \mathrm{mM} \mathrm{pH7.4)} \mathrm{was} \mathrm{prepared} \mathrm{using}$ $200 \mathrm{~mL}$ of ultrapure water adding a tablet of PBS. Artificial sweat was prepared according to the composition in [20].

2) Electrode functionalization for lactate measurements: SPE from Metrohm was used: WE $(4 \mathrm{~mm} \varnothing$ in Pt), CE (Pt), RE (Ag). Several layers of Nafion (5\%) were deposited on the Pt-WE surface. First, a Nafion membrane was realized by three succesive depositions of $1 \mu \mathrm{L}$ Nafion solution, and waiting $20-25$ min between each deposition. LDO stock solution was prepared by dissolving $2.21 \mathrm{mg}$ of LDO in $10.7 \mu \mathrm{L}$ of ultrapure water. $2 \mu \mathrm{L}$ of the stock solution was dropped on the Pt-Nafion WE surface. The sensor was kept at $4^{\circ} \mathrm{C}$, in humid atmosphere, overnight [21].

3) Electrode functionalization for lithium ions measurements: the membrane cocktail for Lithium ions measurement was prepared by dissolving $100 \mathrm{mg}$ of mixture consisting of $28.00 \mathrm{wt} \%$ Poly(vinyl chloride) high molecular weight, $1 \mathrm{wt} \% \mathrm{Li}$ Ionophore VI (6,6-Dibenzyl-1,4,811-tetraoxacyclotetradecane), $0.7 \mathrm{wt} \%$, Potassium tetrakis(4chlorophenyl)borate and $70.3 \mathrm{wt} \%$ 2-Nitrophenyl octyl ether in $1 \mathrm{~mL}$ of Tethraydrofuran (THF). A $10 \mu \mathrm{L}$ drop was then dropcasted onto the SPE after electrochemical nanostructuration of the surface, as described in [3].

\section{B. Lactate measurements}

CA measurements were carried out by applying a constant voltage of $650 \mathrm{mV}$, and by adding multiple injections of $100 \mu \mathrm{L}$ of lactate in a $10 \mathrm{~mL}$ PBS every $\sim 8 \mathrm{~min}$. The logarithmic regression of the lactate calibration measurement is evaluated with an Excel tool. The Limit of Detection (LOD) is found to be $7.02 \mathrm{mM}$; it is computed as $\frac{3 \sigma_{\mathrm{b}}}{\mathrm{S}}$, where $\sigma_{\mathrm{b}}$ is the standard deviation of the blank measurement, and $\mathrm{S}$ is the sensitivity.

The sensor was also tested with artificial sweat, following the same procedure. The amperogram is displayed in Fig. 9a. We observe large variations of the current while injecting lactate at low concentrations. Current variations gradually decreases with larger concentrations. This phenomenon is due to the saturation of the enzyme which is typical for such sensor. The calibration curve is reported in Fig. 9b. A lower LOD $(1.43 \mathrm{mM})$ is noticed, with respect to the measurements in PBS.
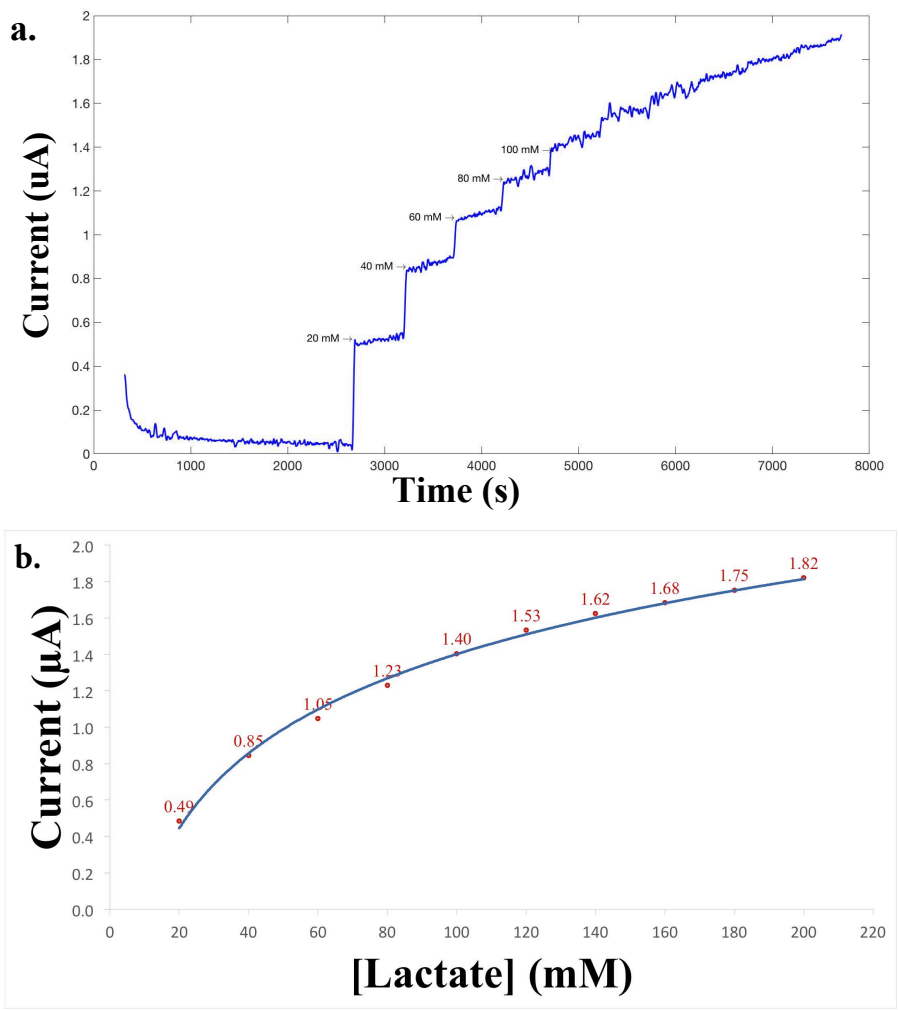

Fig. 9: Lactate detection in sweat: a. Chronoamperometry measurement with $\mathrm{Pt} / \mathrm{Nafion} / \mathrm{LDO}$ electrode, with injection of $20 \mathrm{mM}$ of L-Lactate; $\mathbf{b}$. Calibration curve of Pt/Nafion/LDO electrode: electrode current vs L-Lactate concentration.

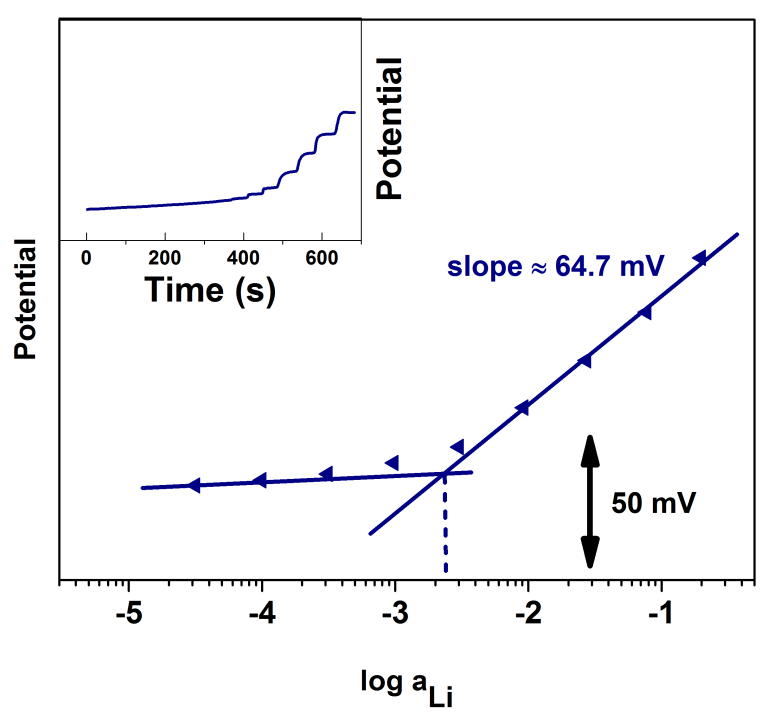

Fig. 10: Lithium calibration in sweat background electrolyte.

\section{Lithium measurements}

An example of a typical time trace obtained from potentiometric measurements performed using a nanostructures-based 
$\mathrm{Li}^{+}-\mathrm{ISE}$ is given in the small inset in Fig. 10. It is evident that sharp and flat potential steps are generated by the addition of increasing amount of lithium ions in solution. From these data, a near-Nernstian calibration curve was obtained, with a slope of $64.7 \mathrm{mV} /$ decade, as shown in Fig. 10. The LOD was calculated, according to IUPAC definition, as the intersection of the extrapolated linear portions of the calibration curve. Its value was found to be around $2.1 \mathrm{mM}$.

\section{Conclusions}

The design and realization of a flexible electrochemical system for combined amperometric and potentiometric measurements has been presented in this work. We have also developed sensing platforms for the detection of lactate and lithium, that are relevant compounds for physical condition monitoring of athletes. The system supports four multiplexed channels: two amperometric and two potentiometric. The former implements a control amplifier to drive the electrochemical cell and the readout circuit to collect the faradaic current. The latter consists of buffered and differential circuitry. A fourth order Sallen-Key filter is designed for signal conditioning. A wireless communication is ensured by exploiting a BLE module. The board has been electrically characterized showing a power consumption of $200 \mathrm{~mW}$, with $3.6 \mathrm{~V}$ power supply. Its flexible substrate and dimensions $(97 \times 66 \mathrm{~mm})$ fit in an armband case, suitable for wearable applications. The hardware system is designed to be interfaced with electrochemical sensors that has been tested for lactate and lithium detection in sweat, with LODs of $1.43 \mathrm{mM}$ and $2.1 \mathrm{mM}$, respectively. The next step will be the integration of the custom-built FPCB with the developed sensors in order to thoroughly validate the system, for sport monitoring applications.

\section{ACKNOWLEDGEMENT}

This research is supported by H2020 ERC 2014 ADG 669354 CyberCare.

\section{REFERENCES}

[1] A. Turner, "Biosensors: Then and now," Trends in Biotechnology, vol. 31, no. 3, pp. 119-120, 2013.

[2] C. L. Reardon and R. M. Factor, "Sport psychiatry," Sports Medicine, vol. 40, no. 11, pp. 961-980, 2010.

[3] F. Criscuolo, I. Taurino, F. Stradolini, S. Carrara, and G. De Micheli, "Highly-stable $\mathrm{Li}+$ ion-selective electrodes based on noble metal nanostructured layers as solid-contacts," submitted to Analytica Chimica Acta, 2018.

[4] K. Mitsubayashi, M. Suzuki, E. Tamiya, and I. Karube, "Analysis of metabolites in sweat as a measure of physical condition," Analytica chimica acta, vol. 289, no. 1, pp. 27-34, 1994.

[5] R. C. Stern, "The Diagnosis of Cystic Fibrosis," The New England Journal of Medicine, vol. 336, no. 7, pp. 487-491, 1997.

[6] D. B. Speedy, T. D. Noakes, and C. Schneider, "Exercise-associated hyponatremia: A review," Emergency Medicine, vol. 13, no. 1, pp. 17$27,2001$.

[7] K. RC, W. KD, S. ML, and et al, "Changes in bone mineral content in male athletes: Mechanisms of action and intervention effects," JAMA, vol. 276, no. 3, pp. 226-230, 1996.

[8] A. Muneer, "Staging Models in Bipolar Disorder: A Systematic Review of the Literature." Clinical psychopharmacology and neuroscience : the official scientific journal of the Korean College of Neuropsychopharmacology, vol. 14, no. 2, pp. 117-30, 2016.
[9] D. F. Smith, "The effect of exercise and pain-stress on the renal clearance of lithium, sodium, potassium and creatinine in the rat," International pharmacopsychiatry, vol. 8, pp. 217-220, 1973.

[10] A. J. Bandodkar, D. Molinnus, O. Mirza, T. Guinovart, J. R. Windmiller, G. Valdés-Ramírez, F. J. Andrade, M. J. Schöning, and J. Wang, "Epidermal tattoo potentiometric sodium sensors with wireless signal transduction for continuous non-invasive sweat monitoring," Biosensors and Bioelectronics, vol. 54, pp. 603-609, 2014.

[11] N. J. Ronkainen, H. B. Halsall, and W. R. Heineman, "Electrochemical biosensors," Chemical Society Reviews, vol. 39, no. 5, p. 1747, 2010.

[12] C. Baj-Rossi, G. De Micheli, and S. Carrara, "A linear approach to multi-panel sensing in personalized therapy for cancer treatment," IEEE Sensors Journal, vol. 13, no. 12, pp. 4860-4865, 2013.

[13] A. J. Bandodkar and J. Wang, "Non-invasive wearable electrochemical sensors: a review," Trends in Biotechnology, vol. 32, no. 7, pp. $363-$ 371, 2014.

[14] D. Sakharov, M. Shkurnikov, M. Y. Vagin, E. Yashina, A. Karyakin, and A. Tonevitsky, "Relationship between lactate concentrations in active muscle sweat and whole blood," Bulletin of experimental biology and medicine, vol. 150, no. 1, pp. 83-85, 2010.

[15] F. Criscuolo, I. Taurino, S. Carrara, and G. De Micheli, "A novel electrochemical sensor for non-invasive monitoring of lithium levels in mood disorders," submitted to IEEE Engineering in Medicine and Biology Society (EMBC'18), 2018.

[16] S. Ghoreishizadeh, C. Baj-rossi, A. Cavallini, S. Carrara, and G. De Micheli, "An Integrated Control and Readout Circuit for Implantable Multi-Target Electrochemical Biosensing," IEEE Transactions On Biomedical Circuits and Systems, vol. 8, no. 6, pp. 1-8, 2014.

[17] F. Stradolini, A. Tuoheti, P. M. Ros, D. Demarchi, and S. Carrara, "Raspberry Pi Based System for Portable and Simultaneous Monitoring of Anesthetics and Therapeutic Compounds," pp. 237-240, 2017.

[18] A. J. Bard and L. R. Faulkner, Electrochemical Methods: Fundamentals and Applications, 2nd ed., J. Wiley, Ed. , 2001.

[19] E. Lindner and R. E. Gyurcsányi, "Quality control criteria for solidcontact, solvent polymeric membrane ion-selective electrodes," Journal of Solid State Electrochemistry, vol. 13, no. 1, pp. 51-68, 2009.

[20] T. Kilic, V. Brunner, L. Audoly, and S. Carrara, "Smart e-patch for drugs monitoring in schizophrenia," in Electronics, Circuits and Systems (ICECS), 2016 IEEE International Conference on. IEEE, 2016, pp. 5760.

[21] N. Patel, A. Erlenktter, K. Cammann, and G.-C. Chemnitius, "Fabrication and characterization of disposable type lactate oxidase sensors for dairy products and clinical analysis," Sensors and Actuators B: Chemical, vol. 67, no. 1, pp. $134-141,2000$. 